\title{
Total gastrointestinal endoscopy in the management of Peutz-Jeghers syndrome
}

\author{
Nicolas De Luca, Yoon Chia, David A Gorard
}

\begin{abstract}
Summary
Peutz-Jeghers syndrome was diagnosed in a 51-year-old woman presenting with iron deficiency anaemia. Upper gastrointestinal endoscopy and colonoscopy revealed several hamartomatous polyps in the stomach, duodenum and colon, which were removed. At a combined surgical-endoscopic procedure, 42 hamartomatous polyps were removed from the small intestine by snare polypectomy. This enteroscopic procedure reduces symptoms, may protect against future intestinal obstructive episodes and their associated surgery, and may reduce the risk of developing gastrointestinal malignancy.
\end{abstract}

Keywords: Peutz-Jeghers syndrome; cancer; enteroscopy

Peutz-Jeghers syndrome (PJS) is a rare autosomal dominant inherited disorder. It is characterised by hamartomatous polyps in the gastrointestinal tract and mucocutaneous melanin hyperpigmentation of the lips, mouth and less commonly the fingers and toes. The melanin spots tend to fade in adult life. ${ }^{12}$ The gene responsible for PJS has been mapped to chromosome $19 \mathrm{p}^{3}$

The polyps in PJS may be multiple or solitary, and are most commonly present in the small intestine (64-96\%), but also occur in the colon $(60 \%)$ and in the stomach $(24-49 \%){ }^{4}$ Patients typically present in the second or third decade of life with infarction, ulceration and bleeding from polyps, resulting in anaemia, or with intestinal obstruction and intussusception.

Before the use of endoscopy, PJS was managed by laparotomy, manual reduction of intussusception, enterotomy, colotomy and surgical polypectomies or segmental resections. ${ }^{5}$ Repeated surgery, which is often necessary in PJS, may be complicated by the formation of peritoneal adhesions and short bowel syndrome. ${ }^{6}$ More recently, intraoperative endoscopy has been used to reduce symptoms from small intestinal polyps and reduce the frequency of emergency surgery. ${ }^{78}$

Between 2-13\% of patients with PJS develop gastrointestinal cancer and a hamartomaadenoma-carcinoma sequence has been suggested. ${ }^{4}$ Other malignant associations are bilateral breast cancer, pancreatic cancer, ovar- ian sex cord tumours, adenoma malignum of the cervix, and feminising Sertoli cell testicular tumours in prepubertal boys. ${ }^{59}$

\section{Case report}

A 51-year-old woman, whose dentist noticed she looked pale, was found to have severe iron deficiency anaemia (haemoglobin $6.9 \mathrm{~g} / \mathrm{dl}$, mean cell volume $63 \mathrm{fl}$ ) and was referred to our gastroenterology clinic. Her only symptoms were intermittent epigastric discomfort and recent increase in bowel frequency.

Her medical history included two episodes of intussusception at age 4, which were managed by laparotomy and manual reduction, appendicectomy at age 16 and mild asthma. At age 33, she required total hysterectomy for a profuse watery vaginal discharge and histology showed adenoma malignum of the cervix. No family history of illness was available, the patient having been adopted, and having no children herself.

Examination was unremarkable except for hyperpigmented spots on the lips (figure 1). Upper gastrointestinal endoscopy revealed three large pedunculated polyps and multiple smaller sessile polyps in the stomach and duodenum. The pedunculated polyps were removed by snare polypectomy. Colonoscopy revealed multiple pedunculated polyps, some up to $4 \mathrm{~cm}$ in size, throughout the colon, which were removed by snare polypectomy. Histology of the gastric and colonic polyps showed these to be hamartomatous polyps of the Peutz-Jeghers type without evidence of malignancy.

Barium follow-through examination revealed at least four large polyps in the mid-jejunum and ileum. To reduce the potential for developing malignancy and

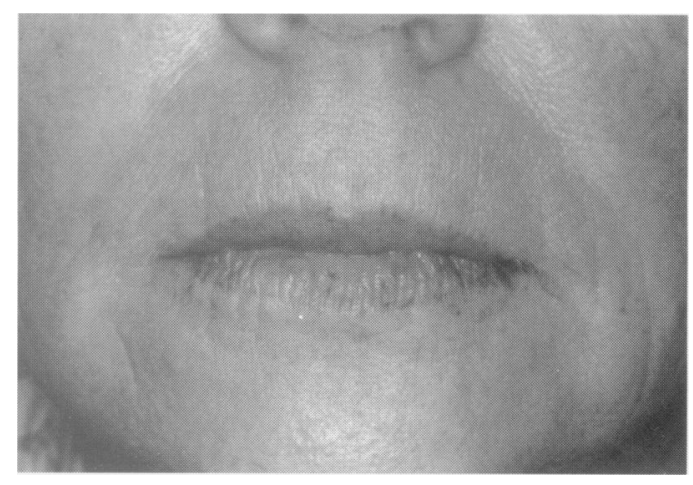

Figure 1 Characteristic hyperpigmented melanin spots of the lips 


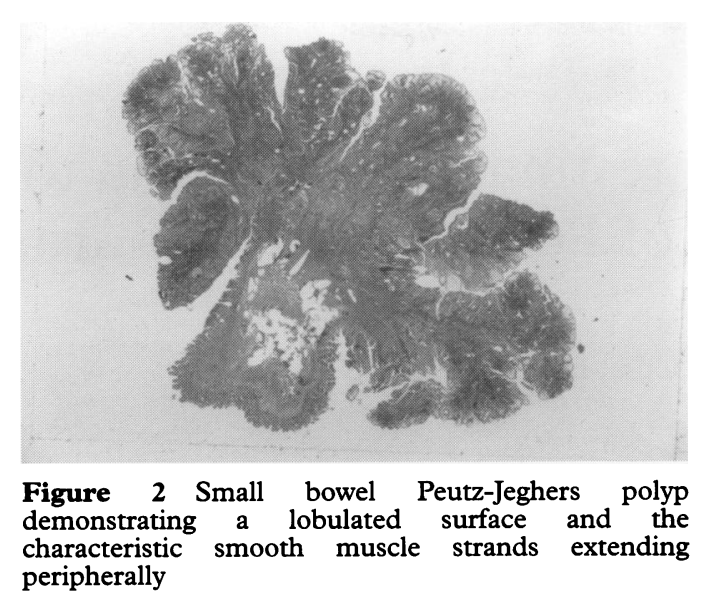

intestinal obstruction in the future, it was decided to remove her small intestinal polyps endoscopically at laparotomy. At laparotomy, the surgeon could palpate approximately 20 polyps throughout the small intestine. A single enterotomy was made in the mid-jejunum at the site of a large polyp. An adult gastroscope, sterilised by 12 hours submersion in glutaraldehyde, was then passed into the small intestine. The endoscope was advanced in a retrograde direction to the duodenum and then antegrade to the terminal ileum with the surgeon helping to concertina the bowel over the gastroscope. A total of 42 polyps, the largest being $3 \mathrm{~cm}$, were snared and removed by cauterisation. The surgeon assisted in retrieval of snared polyps by squeezing the detached polyps towards the enterotomy site. This saved time by eliminating the need to catch every detached polyp with endoscopic retrieval forceps and remove the gastroscope for each polyp. Total operative time was 90 minutes. Histological examination of the small intestinal polyps showed them all to be hamartomatous with no features of malignancy (figure 2).

There were no complications during or after the operation and the patient was discharged home after 7 days. Her anaemia responded to oral iron therapy.

\section{Discussion}

Patients with PJS have gastrointestinal polyposis, most commonly of the small intestine. They are often symptomatic, requiring surgery and there is an increased risk of developing gastrointestinal malignancy as well as other malignancies. Complete endoscopic examination of the gastrointestinal tract and polypectomy allows the patient with PJS a reduction in symptoms of anaemia, intussusception, and intestinal obstruction.

Patients with PJS under the age of 30 years often die of complications secondary to bleeding, intussusception and obstruction. However, patients over 30 years are more likely to die from gastrointestinal malignancy. ${ }^{6}$ There is some evidence for a hamartoma-adenoma-

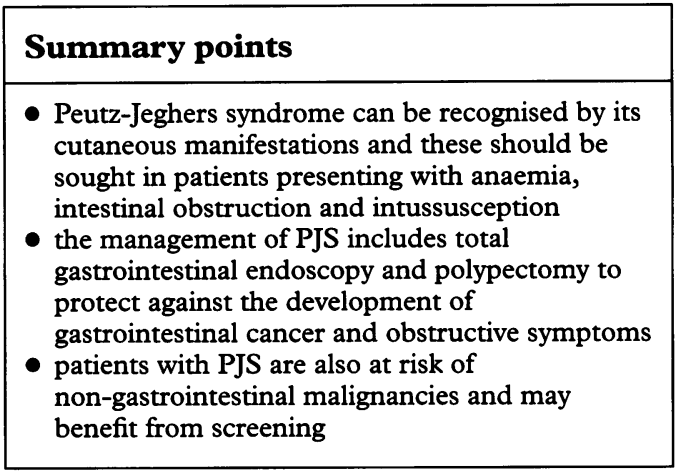

carcinoma sequence, ${ }^{4}$ although it has also been suggested that cancers can develop de novo from normal mucosa. ${ }^{910}$ The small intestine is the most common site for polyps in PJS, but malignancy in the small intestine is much less common than in the colon. An explanation for this may be that large polyps in the small intestine cause obstruction and are removed surgically before they become malignant.

Despite the development of long flexible endoscopes, complete endoscopic examination of the small intestine poses substantial technical difficulties, whether the endoscope is passed via the oral or anal route. At colonoscopy, only a short length of terminal ileum may be inspected. Using a 'push' enteroscope inserted orally, the proximal small intestine may be inspected but the ileum is often not reached. Sonde enteroscopy will more reliably reach the terminal ileum but has the disadvantages of being less controllable and without therapeutic capability. The combined surgical-endoscopic approach we used ensures a quick, thorough assessment of the entire small intestine. Small intestinal polyps not seen with barium imaging and not palpable through the bowel wall by the surgeon can be identified. Multiple polyps can be removed safely with the snare and retrieved for histology without the need for multiple enterotomies. However, the intra-operative surgical-endoscopic approach has the drawback of exposing the patient to the potential complications of general anaesthesia and laparotomy.

In addition to treating anaemia from chronic gastrointestinal blood loss, the total clearance of all polyps from our patient's gastrointestinal tract should provide some protection against the future development of gastrointestinal cancer and obstructive symptoms. Regarding future surveillance, some authors recommend performing gastroduodenoscopy, colonoscopy and barium imaging of the small intestine every two years to look for further polyp development. ${ }^{911}$ Breast and gynaecological screening have also been recommended in women. ${ }^{511}$ Although such surveillance and screening programmes may provide benefit and be justified, demonstrating efficacy of such programmes is difficult, given the rarity of PJS. 
1 Peutz JL. A very remarkable case of familial polyposis of the mucous membrane of the intestinal tract and nasopharynx accompanied by peculiar pigmentations of the skin and mucous membrane. Ned Tijdschr Geneeskd 1921;10:134-46. 2 Jeghers $\mathrm{H}$, McKusick VA, Katz KH. Generalized intestinal polyposis and melanin spots of the oral mucosa, lips and digits: a syndrome of dic 1949;241:1031-6.

3 Amos CI, Bali D, Thiel TJ, et al. Fine mapping of a genetic locus for Peutz-Jeghers syndrome on chromosome $19 \mathrm{p}$. Cancer Res 1997;57:3653-6.

4 De Facq L, De Sutter J, De Man M, Van der Spek P, Lepoutre L. A case of Peutz-Jeghers syndrome with nasal polyposis, extreme iron deficiency anemia, and hamartomaadenoma transformation: management by combined surgical and endoscopic approach. Am $\mathcal{f}$ Gastronterol 1995;90:1330-2.

5 Foley TR, McGarrity TJ, Abt AB. Peutz-Jeghers syndrome: a clinicopathologic survey of the 'Harrisburg family' with a 49-year follow-up. Gastroenterology 1988;95:1535-40.
6 Utsunomiya J, Gocho H, Miyanaga T, et al. Peutz-Jeghers syndrome: its natural course and management. fohn Hopkins Med f 1975;136:71-82.

7 Takehara H, Okada A, Nishi M, Masamune K, Komi N. Intra-operative total enteroscopy for the management of Peutz-Jegher's syndrome. Acta Paediatr fap 1992;34:569-

72.

8 Spigelman AD, Thompson JPS, Phillips RKS. Towards decreasing the relaparotomy rate in the Peutz-Jeghers syndrome: the role of peroperative small bowel endoscopy. Brf Surg 1990;77:301-2.

9 Spigelman AD, Murday V, Phillips RKS. Cancer and the Peutz-Jeghers syndrome. Gut 1989;30:1588-90.

10 Hizawa $\mathrm{K}$, Iida $\mathrm{M}$, Matsumoto $\mathrm{T}$, et al. Cancer in Peutz-Jeghers syndrome. Cancer 1993;72:2777-81.

11 Spigelman AD, Arese P, Phillips RKS. Polyposis: the PeutzJeghers syndrome. Br f Surg 1995;82:1311-4.

\title{
Abnormal liver function tests following inadvertent inhalation of volatile hydrocarbons
}

\author{
J R Pyatt, I Gilmore, P A Mullins
}

\begin{abstract}
Summary
The use of aerosols containing volatile hydrocarbons in conditions of poor ventilation can result in accidental overexposure which can cause central nervous system effects and hepatic injury. We present a case in which inadvertent usage of an adhesive spray used to make greeting cards resulted in vague neurological symptoms and abnormal liver function tests both of which fully resolved on discontinuation.
\end{abstract}

Keywords: liver function tests; volatile hydrocarbons; inhalation; neurological symptoms.

Elevated transaminases may occur following ingestion and vapour inhalation of some hydrocarbons. We report a case in which inadvertent inhalation resulted in hepatic injury and review the evidence for a link between the hydrocarbons contained in the spray and the patient's abnormal liver function tests.

\section{Case report}

Liverpool Royal University Hospital, Prescot Street, Liverpool, L7 8XP, UK Department of Cardiology J R Pyatt

P A Mullins

Department of Gastroenterology, I Gilmore

Correspondence to Dr JR Pyatt, 7 Rigby Street, Hindley, Wigan WN2 4BL, UK

Accepted 30 June 1998
A 63-year-old man attended the Accident and Emergency Department complaining of a 2-month history of non-specific malaise and paraesthesia in his left upper limb. Two days prior to his attendance a general biochemical screen and full blood count were performed by a commercial laboratory through a friend. The results showed an elevated serum alkaline phosphatase of $162 \mathrm{IU} / 1$ (normal range 50136); a serum aspartate transaminase of 73 IU/1 (15-37); a serum alanine transaminase of $122 \mathrm{IU} / 1$ (30-65) and a serum gammaglutamyltransferase of $108 \mathrm{IU} / 1(5-85)$. The serum bilirubin, albumin and protein were all normal. The patient had been previously well and was not taking any medication. He was a non-smoker and drank 1-2 units of alcohol weekly. He had never previously drunk alcohol excessively. On examination he appeared well, was not jaundiced or pyrexial and had none of the stigmata of chronic liver disease. No hepatomegaly or splenomegaly were detected and the rest of the general examination was unremarkable. Various investigations were arranged and he was discharged from hospital and followed up 3 weeks later in the out-patient clinic. His serological markers for acute viral hepatitis were negative. His serum ceruloplasmin and ferritin level and thyroid function tests were normal. His auto-antibody screen was negative. His abdominal ultrasound revealed an echobright pattern suggestive of mild fatty change and an incidental $2 \mathrm{~cm}$ gall stone without biliary tract dilation. A plain cervical spine radiograph showed minor degenerative changes only.

A week later the patient's wife, who worked for Age Concern, attended a drug abuse lecture and recognised the potential cause of her husband's symptoms. He was a keen producer of home-made greeting cards and spent up to 3 hours at a time, two to three times per week, in a poorly ventilated garage using $3 \mathrm{M}$ (TM) Spraymount Adhesive. He had been using it regularly for approximately 2 years but because of both a Christmas 'rush' to produce extra cards for a charity and excessive cold weather, this use had increased and with virtually no ventilation. The patient then confessed to symptoms of drowsiness and nausea. After stopping it completely and changing to a paste form of adhesive he felt dramatically better with complete cessation of his symptoms. Repeat liver function tests taken 4 weeks after the first set had returned to normal. 